\title{
Terahertz Electro-Optic Sampling in Crystals with High Natural Birefringence
}

\author{
I.E. Ilyakov $^{1,2,3}$, G.Kh. Kitaeva ${ }^{4}$, B.V. Shishkin ${ }^{1,2}$, and R.A. Akhmedzhanov ${ }^{1}$ \\ ${ }^{1}$ Institute of Applied Physics RAS, Nizhny Novgorod 603950, Russia, i.ilyakov@hzdr.de \\ ${ }^{2}$ N.I. Lobachevsky State University of Nizhny Novgorod, Nizhny Novgorod 603950, Russia \\ ${ }^{3}$ Helmholtz-Zentrum Dresden-Rossendorf, Bautzner Landstr. 400, 01328 Dresden, Germany \\ ${ }^{4}$ M.V. Lomonosov Moscow State University, Faculty of Physics, Moscow 119991, Russia
}

The femtosecond lasers allows one to generate via various nonlinear processes pulsed terahertz wave radiation (0.1-10 $\mathrm{THz})$ with unique characteristics: subpicosecond duration containing only one-two periods of oscillation and extremely wide spectrum. These terahertz pulses are in demand for different applications which is supported also by sensitive optical methods of coherent detection. In recent years, great progress was made in the laser-to-terahertz pulse conversion efficiency using highly nonlinear organic materials and lithium niobate (LN) crystal structures $[1,2]$. In spite of high conversion efficiencies obtained during generation process there is a lack of data about efficient use of this crystals and crystal structures as detectors. The main reason is that their application in the most popular standard electro-optic (EO) detection set up based on Pockels effect [3] is hindered by decoherence of the laser polarization components because of high natural birefringence of these crystals [4]. In this work we perform the detection methods suited to be used with EO crystals with various natural birefringence and test experimentally their applicability with different EO crystals and crystal structures.

When the laser pulse interacts with $\mathrm{THz}$ in $\mathrm{EO}$ crystal not only its polarization ellipticity but also its energy could be changed $[5,6]$. These changes depend on the amplitude and phase of the $\mathrm{THz}$ overlapped with the laser pulse and can be used for terahertz time-domain measurements $[5,6]$. The method based on energy changes can separately measure the energies of the orthogonal polarization components of the laser pulse and, in contrast to techniques based on ellipticity changes, does not require maintaining coherence between the polarization components and thus, can be used with birefringent crystals.

The sensitivity of both the "energy-based" method and the standard "ellipticity-based" one can be improved if the laser pulses with the edge-cut spectrum are used. As we shown theoretically [7, 8] the relative changes of spectral energy density and ellipticity at the frequencies corresponding to the edge-cut of the laser pulse spectrum could be much higher than when a laser pulse with Gaussian spectrum is used. To realize this in experiment an edge filter can be positioned before the EO crystal to produce the high gradient of the spectral energy density of the laser pulse and the laser pulse after interaction with $\mathrm{THz}$ in the EO crystal can be decomposed in the frequency spectrum by a diffraction grating or can be passed through another edge filter transmitting only the opti- cal waves with frequencies corresponding to the slope of the first filter [7, 8].

These techniques were tested in our experiments with a number of crystals [7-10]: with ZnTe and GaP - commonly used for EO sampling, and with birefringent ones - periodically-poled LN (PPLN) and DSTMS crystals. All of them demonstrated an applicability of being used with the suggested methods.

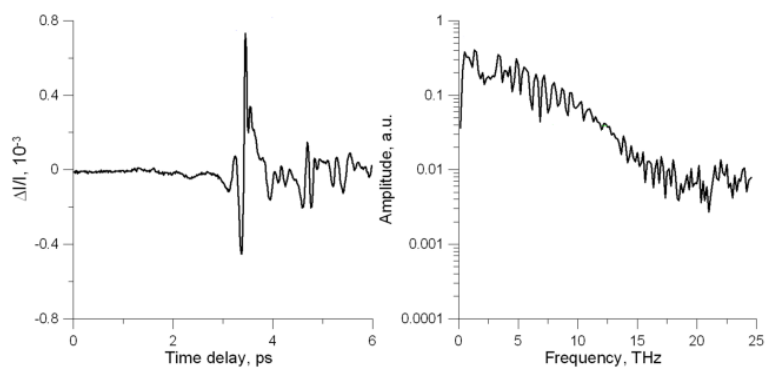

Fig.1. THz wave form and amplitude spectrum measured by DSTMS crystal.

In its easiest configuration the technique based on the total laser pulse energy changes demands even fewer optical elements than the standard one. It was tested as for narrow band detection with PPLN crystal, which allows one to achieve optical-THz synchronism at various terahertz frequencies and laser wavelengths. Application of this method with DSTMS crystal led to significant broadening of the detection bandwidth. The $\mathrm{THz}$ waves with frequencies up to $15 \mathrm{THz}$ could be measured by the laser pulses with duration of $50 \mathrm{fs}$ (Figure 1). However, for the same crystal - ZnTe or GaP this technique demonstrates the lower sensitivity than the standard one.

To improve the sensitivity of measurements the techniques with edge-pass filters were used [7, 8]. At high frequencies they demonstrated more than an order higher sensitivity comparing with the standard detection technique when the same ZnTe and GaP detection crystals were used. Further improvement of the detection sensitivity and bandwidth was achieved with the PPLN (Figure 2) and DSTMS crystals. The use of DSTMS crystal also demonstrated higher bandwidth comparing with the use of GaP crystal.

The sandwich structures with LN core demonstrated previously the high optical-terahertz conversion efficiency [11]. The use of these structures for noncollinear EO sampling was demonstrated previously basing on the spatial modulation of the laser power density [12]. However, due to the less sensitivity of this technique the signal was lower 


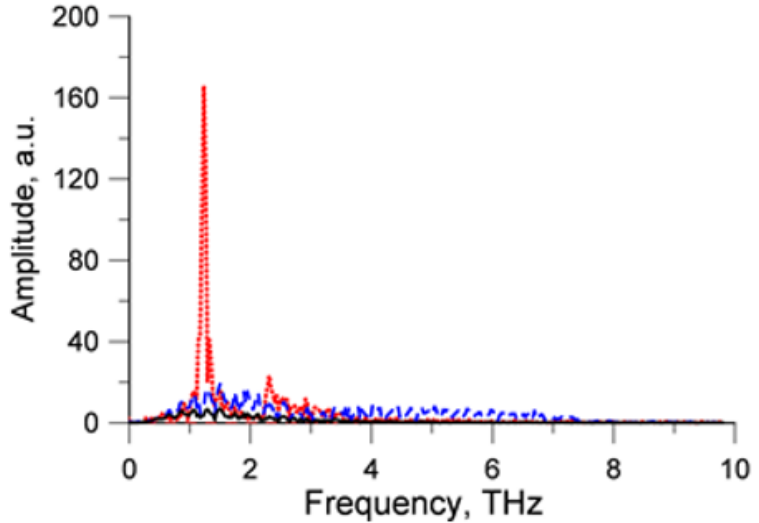

Fig.2. Fourier amplitude spectra of time dependences measured by the standard measurement scheme with the GaP crystal (black-solid line); by the scheme with edge filters and the GaP crystal (blue-dashed line) and with the PPLN crystal (red-dotted line, highest curve).

comparing with the standard one. Our future plan is to combine such structures with the detection methods described above. Due to the noncollinear detection scheme with a $\mathrm{Si}$ prism coupling it is possible to achieve a broadband optical-THz synchronism in LN and reduce the wavelength absorption. The detection sensitivity significantly depends from the probe pulse width. However, for the suggesting method with the edge filters we can take only the part of the optical beam after the crystal and reduce by this way the effective transverse area of the optical- $\mathrm{THz}$ interaction. As is seen from the Figure 3, the detection bandwidth and detection sensitivity could be much higher than the ones of the standard detection technique with the ZnTe crystals of various thicknesses. The calculation results of the detection sensitivity at different probe beam diameters will be also presented in the report.

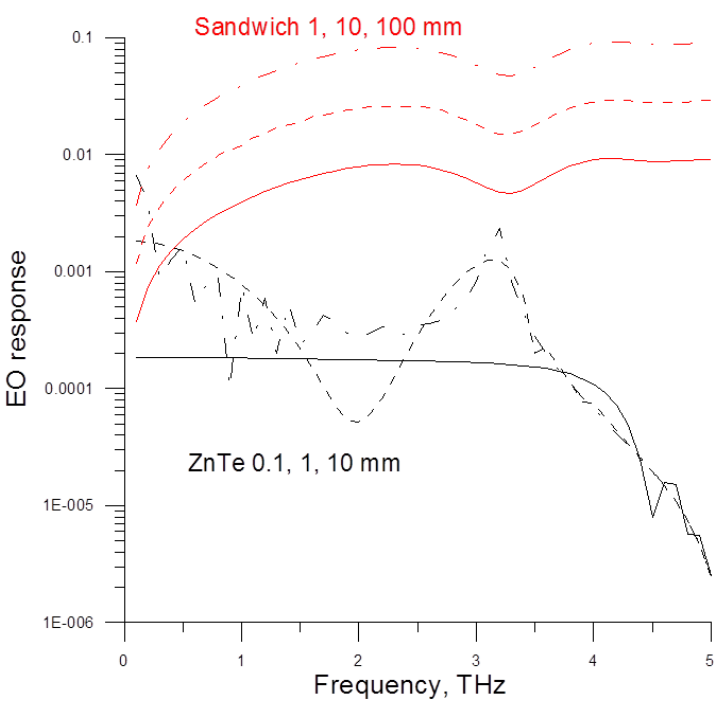

Fig.4. Calculation of the EO response on the same signal of the standard detection technique with the ZnTe crystal and energy detection technique with LN sandwich structure of various lengths (probe beam width $15 \mu \mathrm{m}$ ).

Acknowledgment. This work was supported by the Russian Science Foundation Grant No. 18-1900486.

\section{References}

1. Bodrov, S. B., Ilyakov, I. E., Shishkin, B. V., and Stepanov, $A$. $N$. Efficient terahertz generation by optical rectification in $\mathrm{Si}-\mathrm{LiNbO} 3$-air-metal sandwich structure with variable air gap // Appl. Phys. Lett. 2012. V. 100, N. 20. P. 201114.

2. Vicario, C., Jazbinsek, M., Ovchinnikov, A. V., Chefonov, O. V., Ashitkov, S. I., Agranat, M. B., and Hauri, C. $P$. High efficiency THz generation in DSTMS, DAST and OH1 pumped by Cr:forsterite laser // Opt. Exp. 2015. V. 23. P. 4573-4580.

3. $W u, Q$. and Zhang, $X .-C$. Free-space electro-optic sampling of terahertz beams // Appl. Phys. Lett. 1995. V. 67. P. 3523-3525.

4. Han, P. Y., Tani, M., Pan, F., and Zhang, X.-C. Use of the organic crystal DAST for terahertz beam applications // Opt. Lett. 2000. V. 25. P. 675-677.

5. Kitaeva, G. Kh., Kovalev, S. P., Naumova, I. I., Akhmedzhanov, R. A., Ilyakov, I. E., Shishkin, B. V., and Suvorov E. V. Quasi-phase-matched probe-energy electrooptic sampling as a method of narrowband terahertz detection // Appl. Phys. Lett. 2010. V. 96. P. 071106.

6. Ilyakov, I. E., Kitaeva, G. Kh., Shishkin, B. V., and Akhmedzhanov, R. A. Laser pulse amplitude changes induced by terahertz waves under linear electro-optic effect // Appl. Phys. Lett. 2014. V. 104. P. 151107.

7. Ilyakov, I. E., Kitaeva, G. Kh., Shishkin, B. V., and Akhmedzhanov, $R$. A. Terahertz wave electro-optic measurements with optical spectral filtering // Appl. Phys. Lett. 2015. V. 106. P. 121101.

8. Ilyakov, I. E., Kitaeva, G. Kh., Shishkin, B. V., and Akhmedzhanov, R. A. Terahertz time-domain electro-optic measurements by femtosecond laser pulses with an edge-cut spectrum // Opt. Lett. 2016. V. 41. P. 2998-3001.

9. Ilyakov, I.E., Kitaeva, G.Kh., Shishkin, B.V., and Akhmedzhanov, R.A. Electro-optic sampling of terahertz waves by laser pulses with an edge-cut spectrum in birefringent crystal // Opt. Lett. 2017. V. 42, No. 9. P. 1704-1707.

10. Ilyakov, I.E., Kitaeva, G.Kh., Shishkin, B.V., and Akhmedzhanov, R.A. The use of DSTMS crystal for broadband terahertz electro-optic sampling based on laser pulse amplitude changes // Laser Phys. Lett. 2018. V. 15, No. 12. P. 125401.

11. Bodrov, S. B., Ilyakov, I. E., Shishkin, B. V., and Stepanov, A. N. Efficient terahertz generation by optical rectification in Si-LiNbO3-air-metal sandwich structure with variable air gap // Appl. Phys. Lett. 2012. V. 100, No. 20. P. 201114.

12. Tani, M., Kinoshita, T., Nagase, T., Horita, K., Que, C. T., Estacio, E., Yamamoto, K., and Bakunov, M. I. Non-ellipsometric detection of terahertz radiation using heterodyne EO sampling in the Cherenkov velocity matching scheme // Opt. Exp. 2013. V. 21. P. 9277-9288. 